\title{
Varying expression levels of colony stimulating factor receptors in disease states and different leukocytes
}

\author{
Kyo Young Lee ${ }^{1}$, Byung-Gyu Suh ${ }^{2}$, \\ Jong Wan $\mathrm{Kim}^{2}$, Wonbae Lee ${ }^{2}$, So-Young Kim², \\ Young-Yoo Kim², Jehoon Lee', Jihyang Lim, \\ Myungshin $\mathrm{Kim}^{1}$, Chang Suk Kang ${ }^{1}$ and \\ Kyungja $\operatorname{Han}^{1,3}$ \\ ${ }^{1}$ Department of Clinical Pathology, St. Mary's Hospital, \\ Youngdeungpogu Youidodong 62, Catholic University, \\ Medical College, Seoul 150-713, Korea \\ ${ }^{2}$ Department of Pediatrics, Catholic University Medical College, \\ Seoul 150-713, Korea \\ ${ }^{3}$ Corresponding author: Tel, +82-2-3779-1297; \\ Fax, +82-2-783-6648; E-mail, hankja@cmc.cuk.ac.kr
}

Accepted 29 November 2000

Abbreviations: G-CSF, granulocyte colony stimulating factor; GMCSF, granulocyte macrophage colony stimulating factor; CSFr, receptor for colony stimulating factor

\begin{abstract}
Administration of G-CSF may not always respond in rise of neutrophil counts in different patient population. In order to understand a possible inter-relationship between the G-CSF and GM-CSF induced leukocyte responses and expression levels of receptors for G-CSF (G-CSFr) and GM-CSF (GM-CSFr), the levels of each receptor and CSF were measured in patients with basophilia (8), eosinophilia (14) and bacterial infection showing neutrophilia (12) in comparison with normal healthy adults (12) and children (14). G-CSFr was expressed in neutrophils in the largest amount followed by monocytes, but GMCSFr was expressed more in monocytes than neutrophils. Lymphocytes and basophils did not express G-CSFr or GM-CSFr. The amount of GM-CSFr in neutrophils was present less in patients with infection than normal control $(P=0.031)$. The neutrophils expressed more G-CSFr than GM-CSFr. The quantity of G-CSFr in eosinophil showed marked interval change, higher in acute stage. The plasma concentrations of G-CSF in patients with infection were much higher than normal adults or children $(117.95 \pm 181.16 \mathrm{pg} / \mathrm{ml}, \mathrm{P}<0.05)$. Binding assay with excess amount of CSFs could discriminate the patient who did not show any response to G-CSF or GMCSF administration. After incubation with excess
\end{abstract}

CSFs, more receptors were blocked in children than in adults (G-CSF $P=0.024, G M-C S F P=0.006)$. These results indicate that the amount of CSFr in leukocyte varies in different types of leukocyte, and changes according to the patients' condition even in the same type of leukocyte, and the CSFrs of children bind to CSFs more than those of adults.

Keywords: Granulocyte-colony stimulating factor, granulocyte macrophage-colony stimulating factor, G-CSF receptor, GM-CSF receptor, leukocyte

\section{Introduction}

Granulocyte-colony stimulating factor (G-CSF) and granulocyte macrophage CSF (GM-CSF) are the principal hematopoietic growth factors regulating the production, differentiation and function of granulocytes(Anderlini et al., 1996). To shorten the neutropenic period and the time of engraftment after bone marrow transplantation, CSFs are frequently used in many patients. These CSFs induce granulocyte proliferation by increasing the levels of multiple forms of dihydrofolate reductase, and are mediated through interactions with its receptors (lqbal et al., 2000). Primitive progenitor cells express GM-CSFr (Lund-Johansen et al., 1999) and G-CSFrs are also detected in blast cells of patients with acute myeloid leukemia and acute lymphoblastic leukemia (Shinzo et al., 1997). Although administering G-CSF to a healthy donor does not cause any delayed adverse reaction except a mild bone pain (Sakamaki et al., 1995), in some cases, it can cause a transient increase in blasts mimicking acute leukemia and progressing myelodysplastic syndrome (Meyerson et al., 1999). A few patients do not respond to normal response showing a rapid increase of neutrophil count after administration of G-CSF and mutation in CSFr gene (McLemore et al., 1998) have been implicated as a cause. Diagnosis of G-CSF responders from the non-responders is essential prior to CSF treatment. Expression of receptors for G-CSF (G-CSFr) increases with differentiation in myeloid cells (Shinzo et al., 1995) and the level of high plasma G-CSF correlates with the late engraftment after allogeneic bone marrow transplantation (BMT) (Busch et al., 1998). But the relationship between the expression level of these receptors and the plasma CSF concentration has not been studied. G-CSF and GMCSF administration to patients induce neutrophilia within several hours, but their effects on other types of 
leukocyte are unknown (Tajiri et al., 1997; Sun et al., 1999). Levels of these receptors in different types of leukocytes were measured and correlated with plasma concentrations of CSFs in children, adults and the patients with infection. And also we have developed a new simple binding assay to predict the recovery of neutrophil after CSF administration.

\section{Materials and Methods}

\section{Materials}

EDTA-anticoagulated peripheral blood samples of normal healthy adults (12), children under age of 12 (14), patients with basophilia (8) and eosinophilia (14), and patients with bacterial infection showing neutrophilia (12) were analyzed. Seven out of 8 patients showing basophilia and 2 out of 14 patients showing eosinophilia were diagnosed as chronic myelogenous leukemia. The remaining patient with basophilia had breast carcinoma and 10 remaining patients with eosinophilia showed reactive eosinophilia. All samples were analyzed within $4 \mathrm{~h}$ after collection and maintained at room temperature $\left(18\right.$ to $\left.20^{\circ} \mathrm{C}\right)$ before analysis.

For comparative fluorescence quantification, phycoerythrin (PE) conjugated fluorescence quantification kit (QuantiBRITE, Becton Dickinson, San Diego, CA) and QuantiQuest program were used. PE conjugated antiCSF receptors were purchased from Serotec (UK) and other monoclonal antibodies, PerCP conjugated antiCD19, FITC conjugated anti-CD22 and FITC conjugated anti-CD9, and isotypic control were purchased from Becton-Dickinson (San Diego, CA).

\section{Quantitative analysis of G-CSFr and GM-CSFr}

Immunofluorescence studies were performed using whole blood. All samples were stained with anti-G-CSFr, antiGM-CSFr and with the negative isotypic control antibodies. The samples showing basophilia were stained with anti-CD19, anti-CD22 and anti-CSFrs simultaneously. And the samples showing eosinophilia were stained with anti-CD9 and anti-CSFrs simultaneously. Then the erythrocytes were lysed by incubation in the lysing solution (Becton Dickinson) and the sediments were washed in PBS. Fluorescence was analyzed by flow cytometry (FACSCalibur, Becton Dickinson) using CELLQuest software. Quality control of the flow cytometer was carried out twice a week using CaliBRITE ${ }^{\mathrm{TM}}$ beads (Becton Dickinson) and Autocomp software monthly. Basophils were identified in the lymphocyte gate by their positive staining for FITC-conjugated CD22 monitored in FL1 (log scale), and their negative staining with PerCP-conjugated CD19 monitored in FL3 (log scale) (Figure 2) (Han et al., 1999). Eosinophils were identified in the neutrophil gate by their strong expression of
CD9 (Figure 3) (Wardlaw et al., 1995).

Markers were set using isotypic control sera, so that less than $1 \%$ of cells stained positively. Results were recorded as the geometric mean of gated cells. The mean number of bound PE molecule per cell was calculated using QuantiBRITE and QuantiQuest program.

\section{Quantitative determination of G-CSF and GM-CSF concentrations in plasma}

EDTA-anticoagulated plasma was separated immediately after collection of venous blood, and stored in $-70^{\circ} \mathrm{C}$ freezer until analysis. For the quantitative determination of G-CSF and GM-CSF concentrations in plasma, immunoassay kits ( $R$ \& D Systems, MN, USA) were used according to the manufacturer's instructions.

\section{Binding assay of CSFs to CSFrs}

The binding sites of CSFr to anti-CSFr (Serotec) were blocked by binding of CSF in the preliminary study. To estimate the functional binding sites of CSFrs, the leukocytes were incubated with excess amounts of CSFs for $1 \mathrm{~h}$ at $37^{\circ} \mathrm{C}$. After wash with PBS three times, flow cytometric estimation of CSFrs using monoclonal antibodies was performed as described above. G-CSF (Neutrogen, Choong Wae Pharm, Korea) was added in a concentration of $0.5 \mu \mathrm{g} / 10^{6}$ cells and GM-CSF (Leukogen, LG Pham, Korea) was added in a concentration of $2 \mu \mathrm{g} / 10^{6}$ cells. The differences of the mean number of bound PE molecule per cell before and after incubation in excess amount of CSFs were calculated.

\section{Statistics}

All the statistical data were analyzed using the Chisquare test and Mann-Whitney $U$ test. To evaluate the correlation between the amount of CSFr and plasma CSF concentration, the Pearson correlation coefficient and $P$ value were calculated. The significance was evaluated using Wilcoxon Signed Ranks test using SPSS software.

\section{Results}

\section{Quantity of G-CSFr and GM-CSFr}

G-CSFr was expressed in neutrophils in the largest quantity followed by monocytes in all cases (Table 1). Lymphocytes and basophils did not express G-CSFr (Figure 1,2). No significant difference in the quantity of G-CSFr was found between adults and children or between normal and patients with infection $(P>0.05)$.

GM-CSFr was expressed more in monocytes than neutrophils, but no significant difference was noted between eosinophils and neutrophils $(P=0.546)$ or between eosinophils and monocytes $(P=0.236)$. Lymphocytes and basophils did not express GM-CSFr (Figure 
Table 1. Quantities of receptor molecules for colony stimulating factors in different types of leukocytes in adults, children and patients with infection

(number of bound PE molecules per cell)

\begin{tabular}{llccc}
\hline Receptor & Group & Neutrophils & Monocytes & Lymphocytes \\
\hline G-CSFr & adult $(n=12)$ & $1551 \pm 671$ & $655 \pm 183$ & $55 \pm 27$ \\
& children $(n=14)$ & $1872 \pm 373$ & $914 \pm 311$ & $53 \pm 30$ \\
& infection $(n=12)$ & $1259 \pm 768$ & & $49 \pm 32$ \\
GM-CSFr & adult $(n=12)$ & $742 \pm 224$ & $3129 \pm 1011$ & $42 \pm 29$ \\
& children $(n=14)$ & $831 \pm 55$ & $3288 \pm 852$ & \\
& infection $(n=12)$ & $571 \pm 436$ & &
\end{tabular}

1, 2). No significant difference in the quantity of GMCSFr was found between adults and children ( $P=$ 0.343 ), but the amount of GM-CSFr in neutrophil was less in patients with infection than normal control $(P=$ 0.031). The neutrophils expressed more G-CSFr than GM-CSFr in all cases (Figure 1), and the amount of GCSFr expression was in proportion to that of GM-CSFr expression $(r=0.651, P=0.016)$. The quantities of both CSFrs in neutrophils and monocytes were not related to neutrophil or WBC counts. The monocytes expressed more GM-CSFr than G-CSFr. The quantities of both CSFrs in neutrophil and monocytes were not related to neutrophil or WBC counts. The eosinophils expressed more GM-CSFr than G-CSFr (Figure 3). The quantities of both CSFrs were not related to counts of eosinophils, neutrophil or monocytes $(P>0.05)$ respectively. However, the quantity of G-CSFr in eosinophils showed a marked interval change, higher in acute stage with wide range of expression, in the same patients (Figure 3 ).

\section{G-CSF and GM-CSF concentrations in the plasma} The plasma concentrations of G-CSF and GM-CSF in
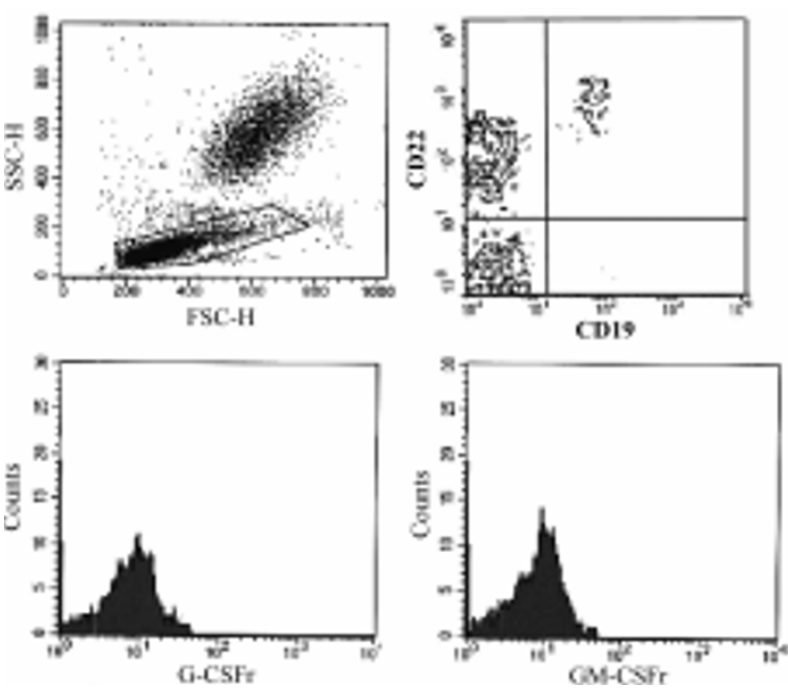

Figure 2. Basophils in the large lymphocyte gate express $C D 22$ without expression of CD19, and they do not express G-CSFr and GM-CSFr on the triple color immunophenotyping.
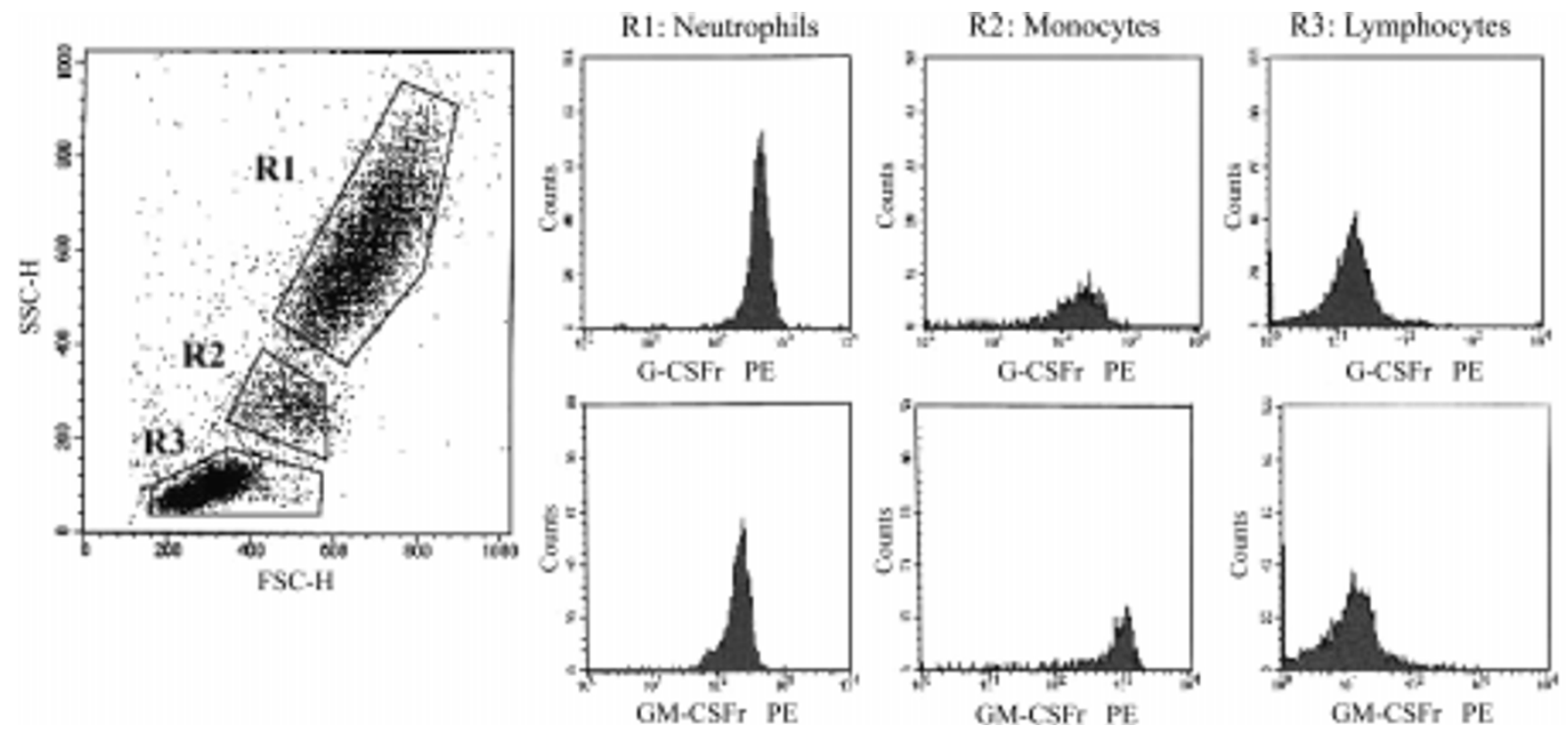

Figure 1. Dot scattergram of leukocytes in the peripheral blood showing neutrophil gate (R1), monocyte gate (R2) and lymphocyte gate (R3) by flow cytometry. Each type of leukocytes shows different quantity of G-CSFr and GM-CSFr on the histograms. 

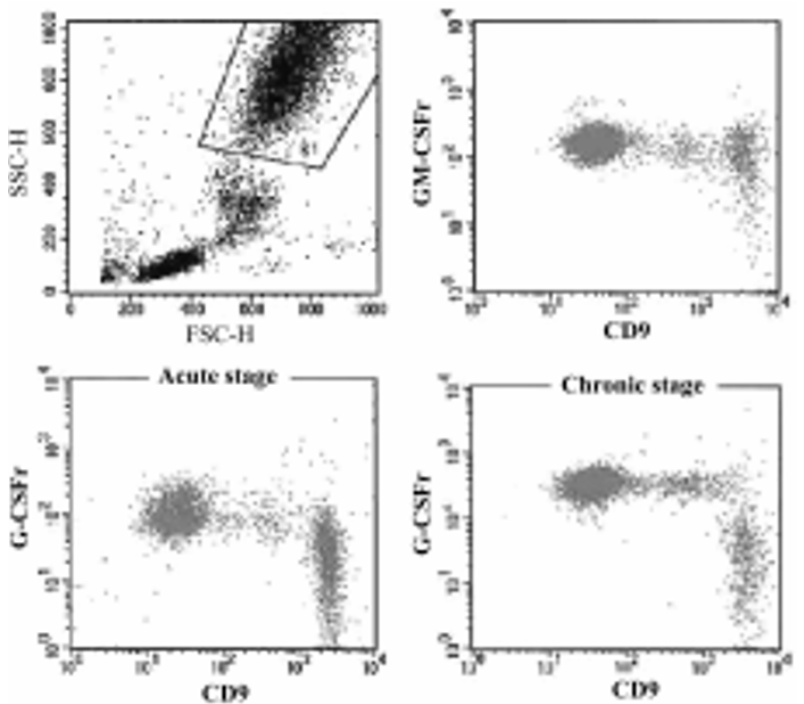

Figure 3. Eosinophils in the neutrophil gate show strong CD9 expression. They reveal similar amount of GM-CSFr and less amount of G-CSFr than the neutrophils. In acute stage of eosinophilia, G-CSFr expression level is higher than in chronic stage in the same patient.

Table 2. The plasma concentration of colony stimulating factors in normal adults, children and patients with infection

\begin{tabular}{lcc}
\hline Group & $\begin{array}{c}\text { G-CSF } \\
(\mathrm{pg} / \mathrm{ml})\end{array}$ & $\begin{array}{c}\text { GM-CSF } \\
(\mathrm{pg} / \mathrm{ml})\end{array}$ \\
\hline Normal adults $(\mathrm{n}=12)$ & $19.42 \pm 0.45$ & $2.44 \pm 0.42$ \\
Normal children $(\mathrm{n}=14)$ & $35.71 \pm 26.79$ & $2.50 \pm 0.51$ \\
Patients with infection $(\mathrm{n}=12)$ & $117.95 \pm 181.16$ & $4.63 \pm 3.22$ \\
\hline
\end{tabular}

normal adults, children and the patients with infection were shown in Table 2. The plasma concentration of GCSF in children was $35.71 \pm 26.79 \mathrm{pg} / \mathrm{ml}$, and it was slightly higher than in adults $(19.42 \pm 0.45 \mathrm{pg} / \mathrm{ml})$ with variations, but the difference was not statistically significant $(P=0.197)$. However, the plasma concentration of G-CSF in patients with infection was much higher than normal adults or children $(117.95 \pm 181.16 \mathrm{pg} / \mathrm{ml}, \mathrm{P}<$ $0.05)$. The plasma concentration of GM-CSF was similar in all 3 groups $(P>0.05)$. The plasma concentrations of CSFs were not related to the amounts of CSFr in neutrophil $(P>0.05)$. But the plasma concentrations of G-CSF were in proportion to the amount of G-CSFr in monocytes $(r=0.776, P=0.005)$.

\section{Results of CSFs binding assay to CSFrs}

Less anti-CSFr molecules were bound to the cells after incubation with excess amount of CSFs in all cases except the patient who did not show any response to GCSF or GM-CSF administration. The patient who did not show any response to G-CSF or GM-CSF administration showed the same amount of CSFr expression in leukocytes before and after incubation in excess amount of CSFs. After incubation with excess CSFs, more re- ceptors were blocked in children than in adults (G-CSF $P=0.024$, GM-CSF $P=0.006$ ).

\section{Discussion}

The levels of G-CSFr and GM-CSFr in different types of leukocytes are not clearly understood. We have found that G-CSFr was expressed largely in neutrophils followed by monocytes and eosinophils in all cases, and GMCSFr was expressed more in monocytes than neutrophils. The lymphocytes and basophils did not express CSFrs. Gessler et al. (1999) demonstrated that less number of neutrophils expressed G-CSFr in neonates than in adults. However, in this study, all neutrophils express G-CSFr and the neutrophils of children showed more CSFrs than adults, although it was not statistically significant. The amount of GM-CSFr in neutrophils was less in patients with infection than normal control $(P=$ 0.031 ) and the amount of G-CSFr in neutrophils showed similar results with the amount of GM-CSFr, although statistically not significant $(1551 \pm 671$ vs. $1259 \pm 768)$. This could be due to the presence of less maturated granulocytes in the peripheral blood of patients with infection. G-CSFrs are expressed in myeloid cells from a very early stage of differentiation and the level of expression increases with the progression of cell maturation (Shinzo et al., 1997). However, the amount of GMCSFr in neutrophil has not been studied previously. In this study, the neutrophils expressed more G-CSFr than GM-CSFr in all cases, and the amount of G-CSFr expression was in proportion to the amount of GM-CSFr expression $(r=0.651, P=0.016)$. And the monocytes expressed more GM-CSFr than G-CSFr. The comparative quantities of CSFrs in eosinophils, basophils and lymphocytes are not known. In this study, the eosinophils expressed more GM-CSFr than G-CSFr like monocytes. GM-CSF is one of the eosinophilopoietic cytokines implicated in allergy in general and function through cell surface receptors (Sun et al., 1999). And interestingly, the quantity of G-CSFr in eosinophils showed marked interval change, higher in acute stage with wide range of expression, in the same patients. This finding means the quantity of CSFr in leukocytes is not constant all the time, but varies according to the patients' condition. The basophils share several characteristics with lymphocytes including scattergram on flow cytometry and expression of CD22 (Han et al., 1999). In this study, the basophils do not express G-CSFr and GM-CSFr as in lymphocytes showing an another same characteristic. The plasma concentration of G-CSF in children was slightly higher with no statistical significance, and more variable in children than in adults (35.71 \pm 26.79 vs. $19.42 \pm 0.45$ $\mathrm{pg} / \mathrm{ml}$ ). Although the quantity of G-CSFr and GM-CSFr in neutrophil was not different significantly, the plasma concentration of G-CSF in patients with infection was 
much higher than normal adults or children as described in previous report (Kawakami et al., 1990). Serum GCSF levels are not increased in patients with antibody induced neutropenia unless they are suffered from infectious diseases (Bux et al., 1999). It indicates that an increased production of G-CSF may be primarily due to the result of infection but not neutropenia.

The plasma concentration of GM-CSF was similar in all 3 groups $(P>0.05)$.

G-CSF treatment has been reported to up-regulate GCSFr in neutrophils (Hustinx et al., 1998). But in this study, the plasma concentrations of CSFs were not related to the amount of CSFr on the neutrophil $(\mathrm{P}>$ 0.05). Instead, the plasma concentrations of G-CSF were in proportion to the amounts of G-CSFr in monocytes $(r=0.776, P=0.005)$. It is possible that $G-C S F$ treatment up-regulates G-CSFr in monocytes.

Binding assay using excess amounts of CSF could discriminate the patients who do not show increase of neutrophil after administration of CSF. G-CSFr deficient mice have chronic neutropenia as in G-CSF deficient mice (Lieschke et al., 1994) and do not expect to have neutrophilia after administration of chemoattractant (Betsuyaku et al., 1999). It means that G-CSFr is important not only in induction of neutrophilia but also in their function. In vitro studies have demonstrated that G-CSF can reverse HIV-associated granulocyte dysfunction (Crawford et al., 1991; Trillet-Lenoir et al., 1993). Therefore, this simple and easy method could predict not only neutrophil recovery after CSF administration, but also responsiveness of G-CSF in granulocyte dysfunction. After an incubation with excess amounts of CSFs, more receptors were blocked in children than in adults (GCSF $P=0.024$, GM-CSF $P=0.006$ ). It is possible that children have more functional CSFrs than adults.

In conclusion, the expressed levels of G-CSFr and GM-CSFr in human leukocytes are different according to the types of leukocytes and even in the same type of leukocytes, it changed according to the patients' condition including age and infection. The CSFrs of children bind to CSFs more than those of adults, and a simple binding assay is recommended to predict the neutrophil recovery after CSF administration.

\section{References}

Anderlini, P., Przepiorka, D., Champlin, R. and Korbling, M. (1996) Biologic and clinical effects of granulocyte colonystimulating factor in normal individuals. Blood 88: 2819-2825

Betsuyaku, T., Leu, F., Semior, R. M., Haug, J. S., Brown, E. J., Jones, S. L., Matsushima, K. and Link, D. C. (1999) A functional granulocyte colony-stimulating factor receptor is required for normal chemoattractant-induced neutrophil activation. J. Clin. Invest. 103: 825-832
Busch, F. W., Pilgrim, T. B., Kramer, A. and Ehninger, G. (1998) Plasma levels of granulocyte colony-stimulating factor in patients after allogeneic bone marrow transplantation for chronic myeloid leukemia correlate with engraftment of transplanted marrow. Bone Marrow Transplant. 19: 653-659

Bux, J., Hofmann, C. and Welte, K. (1999) Serum G-CSF levels are not increased in patients with antibody-induced neutropenia unless they are suffering from infectious diseases. Br. J. Haematol. 105: 616-617

Crawford, J., Ozer, H. and Stoller, R. (1991) Reduction by granulocyte-colony stimulating factor of fever and neutropenia induced by chemotherapy in patients with small cell lung cancer. N. Eng. J. Med. 315: 164-170

Gessler, P., Neu, S., Nebe, T. and Speer, C. P. (1999). Granulocyte colony-stimulating factor receptor expression on neutrophils of term and preterm neonates with and without signs of infection. Eur. J. Pediatr. 158: 497-500

Han, K., Kim, Y., Lee, J., Lim, J., Lee, K. Y., Kang, C. S., Kim, W. I., Kim, B. K., Shim, S. I. and Kim, S. M. (1999) Human basophils express CD22 without expression of CD19. Cytometry 37: 178-183

Hustinx, W. N., Van Kessel, C. P., Heezius, E., Burgers, S., Lammers, J. W. and Hoepelman, I. M. (1998) Effects of granulocyte colony-stimulating factor (G-CSF) treatment on granulocyte function and receptor expression in patients with ventilator-dependent pneumonia. Clin. Exp. Immunol. 112: 334-340

Iqbal, M. P., Burney, I. A., Sultana, F., Mehboobali, N. and Siddiqui T. (2000) Increased levels of multiple forms of dihydrofolate reductase in peripheral blood leukocytes of cancer patients receiving haematopoietic colony-stimulating factors: Interim analysis. Exp. Mol. Med. 32: 84-87

Kawakami, M., Tsutsumi, H., Kumakawa, T., Abe, H., Hirai, M., Kurosawa, S., Mori, M. and Fukushima, M. (1990) Levels of serum granulocyte colony-stimulating factor in patients with infections. Blood 76: 1962-1964

Lieschke, G. J., Grail, D., Hodgson, G., Metcalf, D., Stanley, E., Cheers, C., Fowler, K. J., Basu, S., Zhan, Y. F. and Dunn, A. R. (1994) Mice lacking granulocyte colony-stimulating factor have chronic neutropenia, granulocyte and macrophage progenitor cell deficiency, and impaired neutrophil mobilization. Blood 84: 1737-1746

Lund-Johansen, F., Houck, D., Hoffman, R., Davis, K. and Olweus, J. (1999) Primitive human hematopoietic progenitor cells express receptors for granulocyte-macrophage colonystimulating factor. Exp. Hematol. 27: 762-772

McLemore, M. L., Poursine-Laurent, J. and Link, D. C. (1998) Increased granulocyte colony-stimulating factor responsiveness but normal resting granulopoiesis in mice carrying a targeted granulocyte colony-stimulating factor receptor mutation derived from a patient with severe congenital neutropenia. J. Clin. Invest. 102: 483-492

Meyerson, H. J., Farhi, D. C. and Rosenthal, N. S. (1999) Transient increase in blasts mimicking acute leukemia and progressing myelodysplasia in patients receiving growth factor. Am. J. Clin. Pathol. 109: 675-681 
Sumio, S., Takuya, M., Yasuo, H., Takashi, K. and Yoshiro N. (1995) Haematological study of healthy volunteers 5 years after G-CSF. Lancet 346: 1432-1433

Shinjo, K., Takeshita, A., Ohnishi, K. and Ohno, R. (1995) Expression of granulocyte colony-stimulating factor receptor increases with differentiation in myeloid clls by a newlydevised quantitative flow-cytometric assay. Br. J. Haematol. 91: $783-794$

Shinjo, K., Takeshita, A., Ohnishi, K. and Ohno, R. (1997) Granulocyte colony-stimulating factor receptor at various defferentiation stages of normal and leukemic hematopoietic cells. Leukem. Lymph. 25: 37-46

Sun, Q., Jones, K., McClure, B., Cambareri, B., Zacharakis, B., Iversen, P. O., Stomski, F., Woodcock, J. M., Bagley, C. J., D'Andrea, R. and Loez, A. F. (1999) Stimultaneous antagonism of inerleukin-5, granulocyte-macrophage colony- stimulating factor, and interleukin-3 stimulation of human eosinophils by targetting the common cytokine binding site of their receptors. Blood 94: 1943-1951

Tajiri, J., Noguchi, S. and Murakami, N. (1997) Usefulness of granulocyte count measurement four hours after injection of granulocyte colony-stimulating factor for detecting recovery from antithyroid drug-induced granulocytopenia. Thyroid 7: 575-578

Trillet-Lenor, V., Green, J. and Manegold, C. (1993) Recombinant granulocyte-colony stimulating factor reduces the infectious complications of cytotoxic chemotherapy. Eur. J. Cancer 29: 319-324

Wardlaw, A. J. and Kay, A. B. (1995) Eosinophils:production, biochemistry, and function. In: Beutler E, Lichtman MA, Coller BS, Kipps TJ, editors. Williams Hematology $5^{\text {th }}$ International edition: McGraw-Hill, 799 